\title{
Calculation of interval damping ratio under uncertain load in power system
}

\author{
J. $\mathrm{XING}^{1 *}$, C. $\mathrm{CHEN}^{2}$, and P. WU ${ }^{3}$ \\ ${ }^{1}$ Shanghai Electric Power Design Institute Co., Ltd, 310 South Chongqing Rd., Luwan District, 200025 Shanghai, P. R. China \\ ${ }^{2}$ Dept of Electrical Engineering, Shanghai Jiao Tong University, 800 Dongchuan Rd., Minhang District, Shanghai, China \\ ${ }^{3}$ Songjiang Power Supply Company, Shanghai Municipal Electric Power Company, Shanghai, China
}

\begin{abstract}
The problem of small-signal stability considering load uncertainty in power system is investigated. Firstly, this paper shows attempts to create a nonlinear optimization model for solving the upper and lower limits of the oscillation mode's damping ratio under an interval load. Then, the effective successive linear programming (SLP) method is proposed to solve this problem. By using this method, the interval damping ratio and corresponding load states at its interval limits are obtained. Calculation results can be used to evaluate the influence of load variation on a certain mode and give useful information for improvement. Finally, the proposed method is validated on two test systems.
\end{abstract}

Key words: power system, low frequency oscillation, interval load, interval damping ratio, successive linear programming method, optimization model.

\section{Introduction}

The small-signal stability analysis is mainly focused on low frequency electromechanical oscillations within the range of $0.1-2.0 \mathrm{~Hz}$, which are poorly damped. For a long time, many studies such as modal analysis, controller design [1-2] and algorithm development [3-4] have been conventionally based on deterministic operation parameters. However, the power system is affected by various uncertain factors all the time [5], so that the operation state is fluctuant within a certain range. It is of practical importance to determine the influence of operation state variation on system low frequency oscillation.

Load uncertainty, which is one of the most important uncertainty factors in a network, has great influence on a power system and should be considered in the small-signal stability analysis. At present, some research are developed in the field of small-signal stability taking account of load uncertainty. K.W. Wang and others [6-9] studied the probabilistic eigenvalue analysis method when the load is uncertain. In their studies, assuming normal distribution, normal operation values of load powers are used as the corresponding means.

The interval distribution model, which is a general model to describe uncertain information, just needs to know the upper and lower limits of uncertain information. The theory of interval mathematics has some development in recent years and has been partly applied to research of power system [10-12].

In [13-14], power system damping variation due to the interval uncertainty of load characteristic parameters, is investigated by using a special sampling method which samples limited number of points within interval limits of load pa- rameters. However, the active and reactive steady-state load magnitudes are considered to be invariable in these papers. In fact, the uncertainty of load magnitude changes an operation condition of the system and thus the oscillation modes.

On the background, this paper proposes an analysis method for small-signal stability under interval load. The paper is organized as follows: In Sec. 2, the interval damping ratio optimization model is built, by which the upper and lower limits of interval damping ratio under interval load can be solved. In Sec. 3, the principle of successive linear programming (SLP) method applied to this problem is introduced. In Sec. 4, the SLP model for interval damping ratio optimization is formulated and the procedure of algorithm is given. In Sec. 5, two numerical examples are used to test the proposed methodology. Finally, conclusions are given in Sec. 6.

\section{The interval damping ratio optimization model}

2.1. Definition of interval damping ratio. A power system is a non-linear self-consistent system. The eigenvalues of the linearized system depend on the steady-state operating point. Each eigenvalue is a function of power system operation variables. We assume that $\lambda_{k}$ and $\zeta_{k}$ are the $k$-th eigenvalue and its damping raito. With the variation of loads in their interval ranges, system eigenvalues and damping ratios also change in their interval ranges. Following the definition of interval load, interval eigenvalue and interval damping ratio are defined as follows.

Interval Eigenvalue: Within the interval load, a certain load condition corresponds to lots of eigenvalues, conjugated eigenpair of them standing for oscillation mode. Any eigen-

*e-mail: sjtuxj@hotmail.com 
value among them changes with load variation. Every eigenvalue submits to an interval distribution under interval load. The distribution is defined as interval eigenvalue, written as $\left[\lambda_{k \text { min }}, \lambda_{k \text { max }}\right]$, where $\lambda_{k \text { min }}$ and $\lambda_{k \text { max }}$ represent the lower limit and upper limit of $\lambda_{k}$, respectively.

It should be interpreted that the ranking of interval eigenvalue, which is not unique, depends on the aim of investigation. For a complex eigenvalue, either its real part or imaginary part can be used to arrange elements of interval. In this paper, we sort the interval distribution of eigenvalue by its damping ratio which is an important index to judge the small-signal stability of system.

Interval damping ratio. The damping ratio range corresponding to the interval eigenvalue is defined as interval damping ratio, written as $\left[\zeta_{k \text { min }}, \zeta_{k a x}\right]$, where $\zeta_{k \text { min }}$ and $\zeta_{k \max }$ are the lower limit and upper limit, respectively. According to this definition, the conjugate complex eigenpair representing an oscillation mode have the same interval damping ratio.

2.2. Optimization model for solving interval damping ratio. According to the definition of interval damping ratio in Subsec. 2.1, the lower and upper limits of interval damping ratio of a certain oscillation mode reflect its weakest and strongest damping within interval load.

For any eigenvalue $\lambda_{k}$, the optimization model to solve its upper or lower limit of interval damping ratio is described as follows,

$$
\max \zeta_{k} / \min \zeta_{k}
$$

s.t.

$$
\begin{gathered}
f\left(X, P_{L}, Q_{L}\right)=0, \\
A\left(X, P_{L}, Q_{L}\right) \varphi_{k}=\lambda_{k} \varphi_{k} \\
\zeta_{k}=-\sigma_{k} / \sqrt{\sigma_{k}^{2}+\omega_{k}^{2}} \\
P_{L \min } \leq P_{L} \leq P_{L \max }, \\
Q_{L \min } \leq Q_{L} \leq Q_{L \max },
\end{gathered}
$$

where $\zeta_{k}$ is the damping ratio of eigenvalue $\lambda_{k}=\sigma_{k}+j \omega_{k}$ ( $\sigma_{k}$ is real part and $\omega_{k}$ is imaginary part); $A$ is the reduced state matrix of linearized model in small-signal stability analysis [15]; $\varphi_{k}$ is the right eigenvector associated with the eigenvalue; $X$ denotes all state variables except load powers, i.e. voltage amplitudes, voltage phases, power angles and so on; $P_{L}$ and $Q_{L}$ are space vector composed by active load and reactive load in system and their dimensions are the number of loads; $P_{L \text { max }}, P_{L \text { min }}$ are the upper and lower limits of interval active load, respectively; $Q_{L \text { max }}, Q_{L \text { min }}$ are the upper and lower limits of interval reactive load, respectively. The objective function (1) is the maximal or minimal damping ratio of eigenvalue, which is selected in order to solve the interval of damping ratio for every oscillation under interval load $\left[P_{L \text { min }}, P_{L \text { max }}\right]$ and $\left[Q_{L \text { min }}, Q_{L \text { max }}\right]$. When the upper and lower limit of the the interval number are solved, the interval number can be ascertained, so the objective function is $\max \zeta_{k}$ or $\min \zeta_{k}$. The power flow constraint is expressed by
Eq. (2). The eigen-relationship is expressed by Eq. (3). Equation (4) is the formula to calculate the damping ratio. The interval constraints of load are presented by Eqs. (5) and (6).

\section{Successive linear programming method}

The problem stated in (1)-(6) is a rather complicated nonlinear programming problem. It is difficult to be solved directly by using conventional nonlinear optimization technique due to its non-convexity and multi-variables. The successive linear programming (SLP) method is adopted to solve this problem.

In SLP method, the original problem is linearized around the current operating state. And then the linearized model is solved to compute new state. This process is successively repeated until the desired objective is achieved [16]. The SLP method has been successfully used to solve the optimal power flow problem in [17-18].

The model based on SLP method is stated in the standard form, given by

$$
\min F(x, u)
$$

s.t.

$$
\begin{gathered}
h(x, u)=0, \\
u_{\min } \leq u \leq u_{\max },
\end{gathered}
$$

where $x$ and $u$ are indirect vector and direct vector, respectively.

Using the first-order Taylor series expansion at the current operating point $\left(x^{(r)}, u^{(r)}\right)$, the original problem (7)-(9) is transformed into the following linear programming (LP) problem [19-20]:

$$
\begin{gathered}
\min \Delta F\left(\Delta x^{(r)}, \Delta u^{(r)}\right) \\
=\left[\frac{\partial F}{\partial x}\right]^{T(r)} \Delta x^{(r)}+\left[\frac{\partial F}{\partial u}\right]^{T(r)} \Delta u^{(r)} \\
{\left[\frac{\partial h}{\partial x}\right]^{T(r)} \Delta x^{(r)}+\left[\frac{\partial h}{\partial u}\right]^{T(r)} \Delta u^{(r)}=0,} \\
u_{\min }-u^{(r)} \leq u \leq u_{\max }-u^{(r)},
\end{gathered}
$$

where $T$ is the symbol of transpose and $r$ in round bracket represents the number of iteration.

This linearization is only accurate for a small variation of the increment. Therefore, $\Delta u^{(r)}$ should be in its bounds, written as:

$$
-\eta^{(r)} \leq \Delta u^{(r)} \leq \eta^{(r)}
$$

where $\eta^{(r)}$ are vector with all positive small components.

The solution of linear programming model (10)-(13) gives the incremental change $\Delta u^{(r)}$ of direct vector, which is added to $u^{(r)}$ to get a new updated vector: $u^{(r+1)}=u^{(r)}+\Delta u^{(r)}$. By solving equality constraints (8) with the new updated vector $u^{(r+1)}$, a new indirect vector $x^{(r+1)}$ is computed. Therefore, a new operating state $\left(x^{(r+1)}, u^{(r+1)}\right)$ is obtained. Under this new state, a new LP model can be formed again. This procedure is successively repeated until the optimum is achieved. In a word, by solving a series of LP models, a sequence of optimal solutions of LP model are obtained, which converge to the optimal solution of original nonlinear problem [16]. 
The basic steps of the SLP algorithm to solve (7)-(9) are summarized as follows:

1) Set initial direct variables $u^{(0)}$ which satisfy inequality constraints (9). Calculate initial indirect variables $x^{(0)}$ by solving Eq. (8). Set the step limit $\eta^{(0)}$ of direct vector increment, contractible coefficient $\beta \in(0,1)$, and user defined tolerance $\varepsilon$. Assume the number of direct variables is $l$. Set iterative number $r=0$.

2) Form the linearized interval damping ratio optimization model (10)-(13) under variables $x^{(r)}$ and $u^{(r)}$.

3) Solve the LP problem (10)-(13) and obtain optimal incremental variables $\Delta u^{(r)}$.

4) Update the direct variables: $u^{(r+1)}=u^{(r)}+\Delta u^{(r)}$. Obtain the new indirect variables $x^{(r+1)}$ with updated variables by solving equality constraint (11).

5) If $F\left(x^{(r+1)}, u^{(r+1)}\right)<F\left(x^{(r)}, u^{(r)}\right)$, then $r=r+1$ and go to step 2).

6) If $F\left(x^{(r+1)}, u^{(r+1)}\right) \geq F\left(x^{(r)}, u^{(r)}\right)$ and $\left|\eta_{j}^{(r)}\right| \geq \varepsilon$ $(j=1,2, \ldots, l)$, then $\eta_{j}^{(r+1)}=\beta \eta_{j}^{(r)}$ and go to step 2).

7) If $F\left(x^{(r+1)}, u^{(r+1)}\right) \geq F\left(x^{(r)}, u^{(r)}\right)$ and $\left|\eta_{j}^{(r)}\right|<\varepsilon$ $(j=1,2, \ldots, l)$, then the optimal solution $x^{(r)}, u^{(r)}$ converge and the program terminates.

\section{The SLP method for solving the interval damping ratio optimization model}

4.1. The SLP model for solving interval damping ratio. According to the procedure of SLP method, the original nonlinear programming model (1)-(6) can be restated as the SLP standard model (7)-(9), where $P_{L}, Q_{L}$ are used as direct variables and $X$ are indirect variables. Indirect variables are decided by direct variables.

The linearization process of original model is summarized in this section.

The linearized objective function (1) is given by

$$
\max \Delta \zeta_{k} / \Delta \zeta_{k}
$$

The power flow constraints represented by Eq. (2) can be written in detail:

$$
\left\{\begin{array}{c}
P_{d i}=P_{g i}-U_{i} \sum_{j=1}^{n} U_{j}\left(G_{i j} \cos \theta_{i j}+B_{i j} \sin \theta_{i j}\right) \\
Q_{d i}=Q_{g i}-U_{i} \sum_{j=1}^{n} U_{j}\left(G_{i j} \sin \theta_{i j}-B_{i j} \cos \theta_{i j}\right)
\end{array}\right.
$$

where $i=1,2, \ldots, n$ except the slack bus ( $n$ is the number of buses) and $\theta_{i j}=\theta_{i}-\theta_{j}$. Just considering the regulation of the generator at slack bus, the linearization of power flow Eq. (2) can be written as follows,

$$
\left[\frac{\partial f}{\partial X}\right]_{X_{0}} \Delta X=\left[\begin{array}{c}
\Delta P_{L} \\
\Delta Q_{L}
\end{array}\right]
$$

where $\left[\frac{\partial f}{\partial X}\right]_{X_{0}}$ is the Jacobian matrix.

The linearization of Eq. (3) yields

$$
\Delta A \varphi_{k 0}+A_{0} \Delta \varphi_{k}=\lambda_{k 0} \Delta \varphi_{k}+\Delta \lambda_{k} \varphi_{k 0}
$$

Premultiplying by the left eigenvector $\psi_{k 0}^{T}$ and noting that $\psi_{k 0}^{T} \varphi_{k 0}=1$ and $\psi_{k 0}^{T} \mathrm{~A}_{0}=\psi_{k 0}^{T} \lambda_{k 0}$, the equation above becomes

$$
\begin{gathered}
\Delta \lambda_{k}=\psi_{k 0}^{T}\left(\left[\frac{\partial A}{\partial X}\right]_{X_{0}} \Delta X\right. \\
\left.+\left[\frac{\partial A}{\partial P_{L}}\right]_{P_{L 0}} \Delta P_{L}+\left[\frac{\partial A}{\partial Q_{L}}\right]_{Q_{L 0}} \Delta Q_{L}\right) \varphi_{k 0} .
\end{gathered}
$$

The elements calculation of $\left[\frac{\partial A}{\partial X}\right]_{X_{0}},\left[\frac{\partial A}{\partial P_{L}}\right]_{P_{L 0}}$ and $\left[\frac{\partial A}{\partial Q_{L}}\right]_{Q_{L 0}}$ can be seen in [21-22].

The damping ratio calculation formula of Eq. (4) can be linearized as follows,

$$
\Delta \zeta_{k}=\left(\sigma_{k 0}^{2}+\omega_{k 0}^{2}\right)^{-\frac{3}{2}}\left(-\omega_{k 0}^{2} \Delta \sigma+\sigma_{k 0} \omega_{k 0} \Delta \omega\right) .
$$

The linearized form of inequality constraints (5)-(6) are

$$
\begin{gathered}
P_{L \text { min }}-P_{L 0} \leq \Delta P_{L} \leq P_{L \max }-P_{L 0}, \\
Q_{L \text { min }}-Q_{L 0} \leq \Delta Q_{L} \leq Q_{L \max }-Q_{L 0} .
\end{gathered}
$$

The bounds on $\Delta P_{L}$ and $\Delta Q_{L}$

$$
\begin{aligned}
& -\eta_{P} \leq \Delta P_{L} \leq \eta_{P}, \\
& -\eta_{Q} \leq \Delta Q_{L} \leq \eta_{Q},
\end{aligned}
$$

where $\eta_{P}, \eta_{Q}$ are the limits of active load and reactive load increment.

Model (14)-(21) is a linear programming model derived from (1)-(6). From Eq. (15), $\Delta X$ can be expressed in terms of $\Delta P_{L}$ and $\Delta Q_{L}$. Substituting the expression for $\Delta X$ in (14), (16) and (17), we obtain the formulation of $\Delta \zeta_{k}$ expressed by $\Delta P_{L}$ and $\Delta Q_{L}$. The equivalent optimization model in the desired final form is

$$
\min c_{P}^{T} \Delta P_{L}+c_{Q}^{T} \Delta Q_{L}
$$

s.t.

$$
\begin{gathered}
P_{L \min }-P_{L 0} \leq \Delta P_{L} \leq P_{L \max }-P_{L 0} \\
Q_{L \min }-Q_{L 0} \leq \Delta Q_{L} \leq Q_{L \max }-Q_{L 0} \\
-\eta_{P} \leq \Delta P_{L} \leq \eta_{P} \\
-\eta_{Q} \leq \Delta P_{L} \leq \eta_{Q}
\end{gathered}
$$

where $c_{P}, c_{Q}$ are coefficients in objective function. When the objective function in (14) is $\max \Delta \zeta_{k}$, it can also be represented by the minimization equation in (22) in form of min $-\Delta \zeta_{k}$.

Due to constraints of optimization model (22) are only the limits of direct vector, the optimal increment of direct vector can be solved by following equations.

$\Delta P_{L j}=\left\{\begin{array}{l}\max \left(P_{L j \min }-P_{L j 0},-\eta_{P j}\right) \text { for } c_{P j} \geq 0 \\ \min \left(P_{L j \max }-P_{L j 0}, \eta_{P j}\right) \text { for } c_{P j}<0\end{array}\right.$

$\Delta Q_{L j}=\left\{\begin{array}{l}\max \left(Q_{L j \min }-Q_{L j 0},-\eta_{Q j}\right) \text { for } c_{Q j} \geq 0 \\ \min \left(Q_{L j \max }-Q_{L j 0}, \eta_{Q j}\right) \text { for } c_{Q j}<0\end{array}\right.$

where $j=1,2, \ldots, l$ ( $l$ is the number of loads). 
4.2. Algorithm to solve the interval damping ratio optimization model. The interval damping ratio optimization model (1)-(6) can be solved by successive linear programming method introduced in Sec. 3. The basic steps to solve the interval damping ratio of eigenvalue are summarized as follows:

Step 1. Set initial load states $P_{L}^{(0)}, Q_{L}^{(0)}$ as initial vector, which satisfy constraints (5) and (6). Compute indirect vector $X^{(0)}$ under given load states by Eq. (2). Then calculate system eigenvalues and their damping ratios. Set the initial increment limits $\eta_{P}^{(0)}, \eta_{Q}^{(0)}$ of $P_{L}^{(0)}$ and $Q_{L}^{(0)}$. Set the contractible coefficient $\beta$, which is a positive number less than unit. Set the user defined tolerance $\varepsilon$.

Step 2. Select the required eigenvalue $\lambda_{k}$ and its damping ratio $\zeta_{k}$. Let iterative number $r$ equal to 0 .

Step 3. Linearize the interval damping ratio optimization model (1)-(6) around the current operation states $X^{(r)}, P_{L}^{(r)}$ and $Q_{L}^{(r)}$. Formulate the linear programming model (14)-(21).

Step 4. Transform model (14)-(21) into the equivalent desired final form (22) and obtain the coefficient vectors of objective function: $c_{P}^{(r)}, c_{Q}^{(r)}$.

Step 5. According to (23) and (24), the incremental changes in the load vector, $\Delta P_{L}^{(r)}$ and $\Delta Q_{L}^{(r)}$, are computed.

Step 6. Update load states: $P_{L}^{(r+1)}=P_{L}^{(r)}+\Delta P_{L}^{(r)}$, $Q_{L}^{(r+1)}=Q_{L}^{(r)}+\Delta Q_{L}^{(r)}$. Solve power flow Eq. (2) to get the new variables $X^{(r+1)}$ with updated load states $P_{L}^{(r+1)}$ and $Q_{L}^{(r+1)}$. Recalculate eigenvalue $\lambda_{k}^{\prime}$ and its damping ratio $\zeta_{k}^{\prime}$.

Step 7. If $\zeta_{k}^{\prime}-\zeta_{k}<0$, let $\zeta_{k}^{\prime}=\zeta_{k}, r=r+1$ and go to step 3 .

Step 8. If $\zeta_{k}^{\prime}-\zeta_{k} \geq 0$, reformulate the LP model (22) with $P_{L}^{(r+1)}, Q_{L}^{(r+1)}$ and $X^{(r+1)}$, in which new coefficient vectors of objective function are $c_{P}^{(r+1)}$ and $c_{Q}^{(r+1)}$.

Step 9. If there is sign-changing between $c_{P j}^{(r)}$ and $c_{P j}^{(r+1)}$ ( $j=1,2, \ldots, l, l$ is the number of direct variables), let $\eta_{P j}^{(r+1)}=\beta \eta_{P j}^{(r)}$; If there is sign-changing between $c_{Q j}^{r}$ and $c_{Q j}^{(r+1)}(j=1,2, \ldots, l, l$ is the number of direct variables), let $\eta_{Q j}^{(r+1)}=\beta \eta_{Q j}^{(r)}$. Else, if $P_{L j}^{(r+1)}=P_{L j \max }$ or $P_{L j}^{(r+1)}=P_{L j \min }$, let $\eta_{P j}^{(r)}=0$; if $Q_{L j}^{(r+1)}=Q_{L j \max }$ or $Q_{L j}^{(r+1)}=Q_{L j \min }$, let $\eta_{Q j}^{(r)}=0$.

Step 10. If $\left|\eta_{P}\right|<\varepsilon$ and $\left|\eta_{Q}\right|<\varepsilon$, the optimum is obtained and output the results, otherwise go to step 3 .

For the procedure above, there are several points which should be interpreted.

Method to set the interval value of uncertain load. The load in power system is always changing. It is difficult to get its precise value. So it is uncertain. In this paper, the interval model is used to describe the uncertainty of load. The interval value of the uncertain load is set by the power system operator according to the practical operation condition of power system. The interval number can be set according to the load value in history, so its upper and lower limits are very easy to be set. The range of the interval can be set wider if the load uncertainty is greater. In this paper, we adopted $\pm 5 \%$ offset of basic states in the numerical examples.

Regulation of increment step. In Step 1, the initial load states $P_{L}^{(0)}$ and $Q_{L}^{(0)}$ are the middle values of their interval distributions.

From the realization process of successive linear programming method, it can be seen that the increment step limits $\eta$ of direct variables are important parameters to judge the algorithm convergence and determine convergent speed, which is dynamically adjusted when solving the optimum.

For example, the initial increment step limits $\eta_{P j}^{(0)}$ is dependent on the initial load value $P_{L j}^{(0)}$ and its interval range. In order to converge quickly, $\eta_{P j}^{(0)}$ is given a big initial value. With the objective value approximating to the optimum, $\eta_{P j}^{(r)}$ should be reduced dynamically. The reduction magnitude is determined by the contractible coefficient $\beta$ whose value is constant and we adopt $1 / 2$ in examples. The detailed procedure of regulation is as follows.

When the objective value increases and the element $c_{P j}^{(r)}$ of vector $c_{P}^{(r)}$ changes its sign, the original direction of related load variation is not at the direction of searching the optimum, because the slope of damping ratio with respect to load is opposite due to the large load increment step. In such case, $\eta_{P j}^{(r)}$ should be reduced to limit the variation step of load, that is $\eta_{P j}^{(r+1)}=\beta \eta_{P j}^{(r)}$.

Nevertheless, if $P_{L j}^{(r+1)}$ reaches its upper limit $P_{L j \max }$ or lower limit $P_{L j \text { min }}$, the increment step limit $\eta_{P j}^{(r)}$ will be of no use, because $P_{L j}^{(r+1)}$ cannot be changed beyond its constraint of interval range. So the value of $\eta_{P j}^{(r)}$ is set to zero.

Selection of objective function. In theory, the objective function can solve the limits of interval damping ratio for any eigenvalue. Generally speaking, we are only interested in several eigenvalues with the weakest damping ratios. Therefore, in Step 6, after recalculating the eigenvalues, the required several smallest damping ratios will be sequenced and selected for the next optimization procedure.

Ccalculation of eigenvalue and eigenvector. In order not to miss eigenvalues, the QR algorithm is used to calculate the eigenvalues. In order to form constraint (16), after calculating the eigenvalues, the inverse power method is used to calculate the eigenvector just for that several selected eigenvalues. In addition, interval damping ratios of two complex conjugate eigenvalues are uniform. So it just needs to solve one of them.

Explanation of the algorithm. Because the realization of algorithm is based on iterative search, the interval damping ratios solved actually exist in system and there are actual operation conditions corresponding to them.

\section{Numerical examples}

The proposed method is tested with two different networks. The program is based on Small Signal Stability Analysis Package (SSAP) developed by Shanghai Jiao Tong University [23]. 
Machine system. In the 4-machine system of Fig. 1, all machines are equipped with controllable rectifier excitations. Parameters of machine are shown in [15]. The operation scenario deterministic load condition is shown in Appendix.

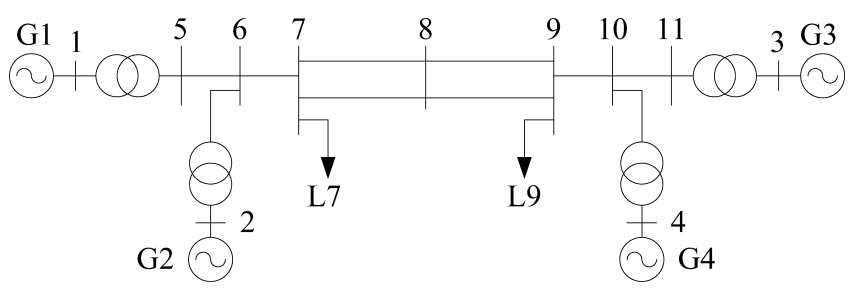

Fig. 1. 4-machine system

With all generators represented by the sixth order machine model, solution under the deterministic load condition gives 36 eigenvalues ( 22 real and 14 complex). 7 pairs of complex eigenvalues have the frequency between $0.1 \mathrm{~Hz}$ and $2.0 \mathrm{~Hz}$, in which electromechanical swings modes can be judged by calculating their electromechanical relative coefficients. In this numerical example, only some important modes' interval damping ratios sorted by frequency are calculated. In fact, all of other modes' interval distribution can be gotten in the same way.

When the load is uncertain, without loss of generality, its interval distributions of active power and reactive power are assumed to be $\left[0.95 P_{L 0}, 1.05 P_{L 0}\right]$ and $\left[0.95 Q_{L 0}, 1.05 Q_{L 0}\right]$, where $P_{L 0}$ and $Q_{L 0}$ are the deterministic load condition in Appendix.

Firstly, under the initial load state $P_{L 0}$ and $Q_{L 0}$, the interval damping ratio optimization model (1)-(6) is established and linearized in the form of (22). Then, taking the five weakest pairs of eigenvalues for example, their interval damping ratios are solved by the proposed SLP method. The calculation results are shown in Table 1 . In the five pairs of modes, the first three ones are electromechanical modes.

Table 1

Interval damping ratio results of 4-machine system

\begin{tabular}{ccc}
\hline \hline Mode & $\begin{array}{c}{\left[\zeta_{\min }, \zeta_{\max }\right]} \\
(\text { The Proposed Method })\end{array}$ & $\begin{array}{c}{\left[\zeta_{\min }, \zeta_{\max }\right]} \\
(\mathrm{MCS})\end{array}$ \\
\hline$\lambda_{1,2}$ & {$[0.022021,0.032179]$} & {$[0.022500,0.031814]$} \\
\hline$\lambda_{3,4}$ & {$[0.103732,0.104264]$} & {$[0.103835,0.104229]$} \\
\hline$\lambda_{5,6}$ & {$[0.135715,0.160957]$} & {$[0.136412,0.160674]$} \\
\hline$\lambda_{7,8}$ & {$[0.603064,0.606813]$} & {$[0.603295,0.606468]$} \\
\hline$\lambda_{9,10}$ & {$[0.617165,0.631879]$} & {$[0.619322,0.630617]$} \\
\hline
\end{tabular}

In order to validate the results of the proposed method, the Monte Carlo Simulation (simply denoted as MCS) method is also used to solve the problem in this paper. MCS is a common method to analyze uncertainty. Through random sampling within the interval load, many samples with deterministic load states can be got and their corresponding damping ratios can be solved. Then, by choosing the maximum and minimum damping ratio of each eigenvalue from simulation results, the interval damping ratio of each eigenvalue can be obtained. The more times the samplings are done, the more precise the interval damping ratio will be. For this 4-machine test system, the sampling times of MCS is 5000. The calculation results are also shown in Table 1.

By comparing the results in Table 1, it is clearly seen that the interval damping ratios solved by the method proposed in this paper are in reasonable ranges. From the realization process of the algorithm, it can be seen that the upper and lower limits of damping ratio resulted from the proposed method corresponds to certain load states, so the results do exist. However, some load states are missed by MCS due to the restriction of sampling times. It is why our interval damping ratios contain the ones form MCS. Comparison results illustrate that the proposed method is effective for the problem.

The optimum of damping ratio is searched by adjusting load along the optimal incremental direction from the initial load condition $P_{L 0}$ and $Q_{L 0}$. Take $\lambda_{1,2}$ with the weakest damping ratio for example to explain the searching procedure in detail, as shown in Fig. 2. The start point at y-axis represents the damping ratio of $\lambda_{1,2}$ when $P_{L}$ and $Q_{L}$ are in the initial deterministic load values $P_{L 0}$ and $Q_{L 0}$. The x-axis stands for the number of iteration. Two curves in figure denote the searching process of damping ratio until the upper and lower limits are reached.

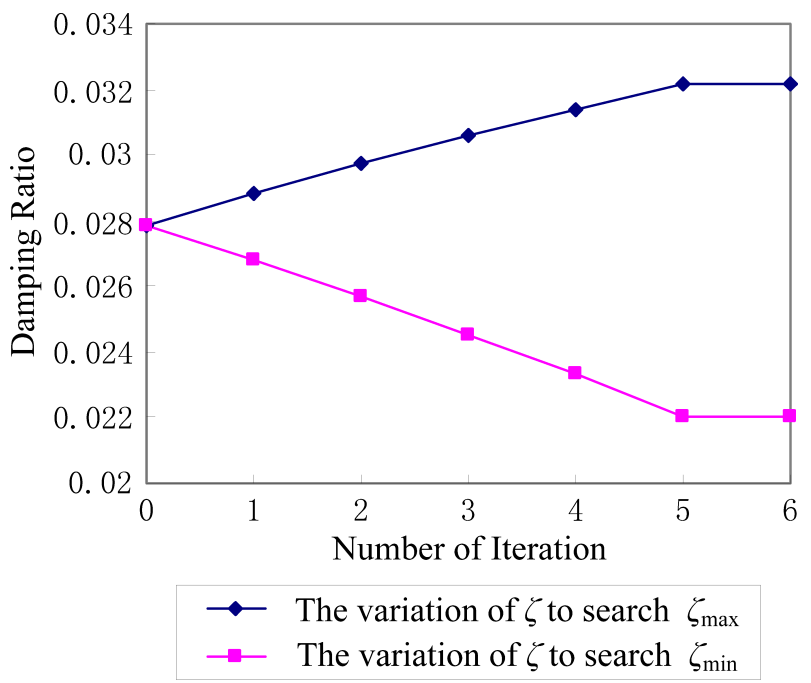

Fig. 2. Searching procedure of the interval damping ratio for 4-machine system

The active load and reactive load are adjusted during each iteration according to the optimization method. The changing procedures are shown in Figs. 3 and 4, separately.

By analyzing the curves in Figs. 2 and 3, we can find that when $P_{L 9}$ rises, the damping ratio of $\lambda_{1,2}$ increases. So the relationship between $\lambda_{1,2}$ and $P_{L 9}$ is monotonic increasing. Similarly, it can be found that the relationship between $\lambda_{1,2}$ and $P_{L 7}$ is monotonically decreasing. 


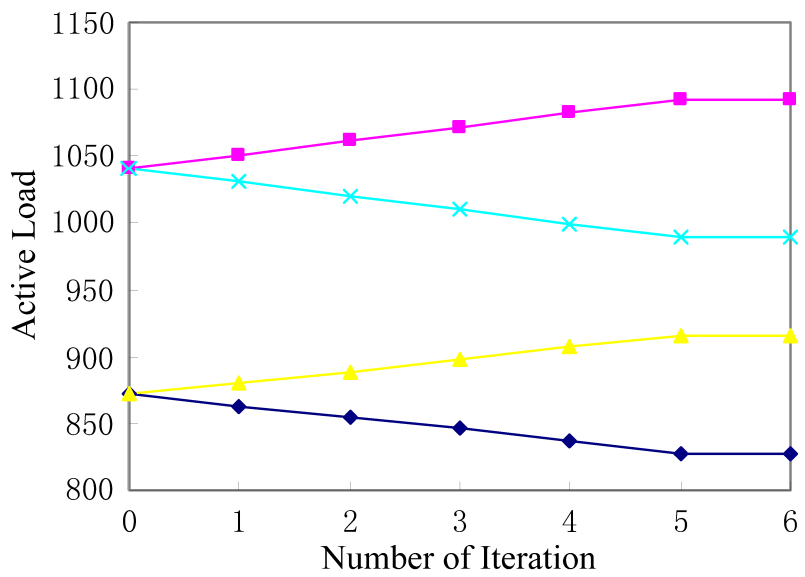

$\longrightarrow$ The variation of $P_{\mathrm{L} 7}$ to search $\zeta_{\max }$

$\rightarrow$ The variation of $P_{\mathrm{L} 9}$ to search $\zeta_{\max }$

The variation of $P_{\mathrm{L} 7}$ to search $\zeta_{\min }$

The variation of $P_{\mathrm{L} 9}$ to search $\zeta_{\min }$

Fig. 3. The changing procedure of active load for 4-machine system

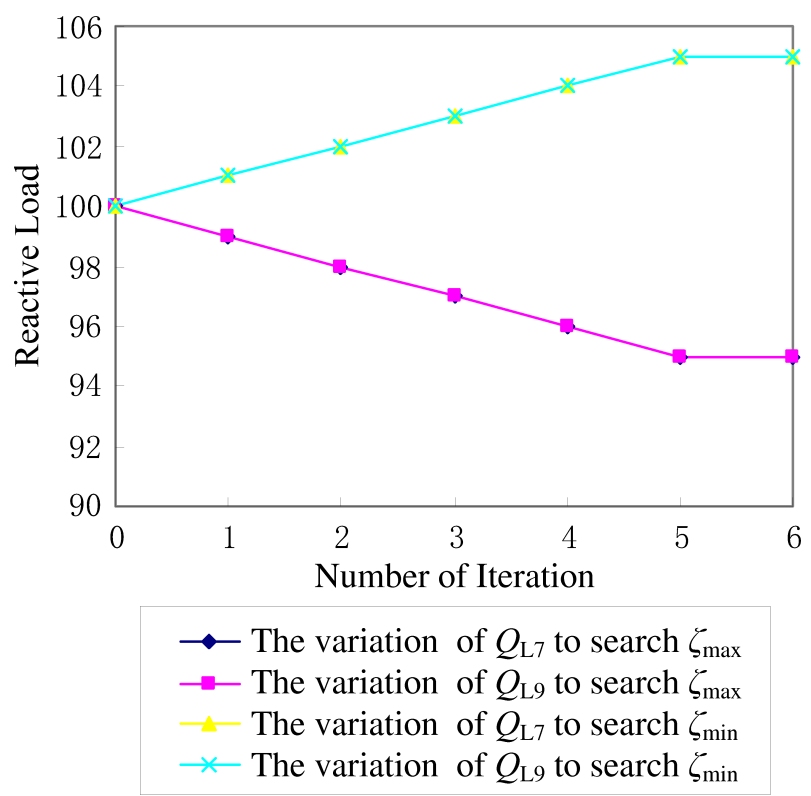

Fig. 4. The changing procedure of reactive load for 4-machine system

The law of damping ratio changing with reactive load is illustrated in Fig. 4. In this example, the initial value and adjusting procedure of the reactive load $Q_{L 7}$ and $Q_{L 9}$ are the same during whole iterations. Therefore the curves of $Q_{L 7}$ and $Q_{L 9}$ for searching the minimal (maximal) damping ratio are superposition.

In Table 2, the load states corresponding to the interval limits of five modes are shown. The first four columns are the load states corresponding to the minimal damping ratio of every mode. The load states corresponding to the maximal damping ratio of every mode are listed in the last four columns.

From Table 2, we can find that the damping ratio limits of different oscillation modes correspond to different load conditions. When the active load or reactive load increase, all damping ratio will not always become worse. For example, when active powers of load 7 and load 9 are at their upper limits, the damping ratios of mode 2 and mode 4 get their maxima. When reactive powers of load 7 and load 9 are at their upper limits, the damping ratios of mode 3 and mode 5 get their maxima. Moreover, the damping ratio of oscillation mode changing with load is not always monotonicity, for example, when the damping ratio of mode 4 is at its minimum, the active load $P_{L 9}$ is not at its limits. When the damping ratio of mode 5 is at its maximum, the active load $P_{L 7}$ is not at its limits.

Table 2

Load states corresponding to the interval limits of 4-machine system

\begin{tabular}{ccccccccc}
\hline \hline \multirow{2}{*}{ Mode } & \multicolumn{9}{c}{$\zeta_{\text {in }}$} \\
\cline { 2 - 9 } & $P_{L 7}$ & $Q_{L 7}$ & $P_{L 9}$ & $Q_{L 9}$ & $P_{L 7}$ & $Q_{L 7}$ & $P_{L 9}$ & $Q_{L 9}$ \\
\hline 1 & 916 & 105 & 989 & 105 & 828 & 95 & 1093 & 95 \\
\hline 2 & 828 & 105 & 1093 & 95 & 916 & 95 & 1093 & 105 \\
\hline 3 & 916 & 95 & 1093 & 95 & 828 & 105 & 989 & 105 \\
\hline 4 & 828 & 95 & 1033 & 95 & 916 & 95 & 1093 & 105 \\
\hline 5 & 916 & 95 & 1093 & 105 & 843 & 105 & 989 & 105 \\
\hline
\end{tabular}

In addition, because the relationship between load power and oscillation mode is nonlinear, the influence of load power variation on oscillation modes is not simple combination of just considering the active load variation or the reactive load variation. In order to estimate the influence of load uncertainty on system oscillation modes correctly, the uncertainty of active and reactive power should be considered at the same time.

The computing time to obtain the interval damping ratio of different mode is listed in Table 3. The second column and the third column give the time to solve the lower limit and upper limit of interval damping ratio for different mode, respectively. The total time to obtain the interval damping ratio is the sum of them, given in the fourth column.

Table 3

Computing time of the interval damping ratio of 4-machine system (unit: second)

\begin{tabular}{cccc}
\hline \hline Mode & Time to get $\zeta_{\min }$ & Time to get $\zeta_{\max }$ & Total Time \\
\hline 1 & 2.56 & 2.57 & 5.13 \\
\hline 2 & 2.57 & 7.22 & 9.79 \\
\hline 3 & 2.31 & 2.57 & 4.88 \\
\hline 4 & 3.58 & 6.19 & 9.77 \\
\hline 5 & 6.16 & 3.33 & 9.49 \\
\hline
\end{tabular}

New England system. The New England system comprising of 10-machine and 39-bus is frequently cited in the small signal stability analysis. In the New England system, all machines are equipped with continuous rotating DC exciters. All generators are represented by the sixth order model. The operating condition is given in Appendix.

The small signal stability analysis under the deterministic load condition gives 110 eigenvalues (54 real and 56 complex). Now, suppose the load is uncertain and the interval distributions of its active power and reactive power are $\left[0.95 P_{L 0}\right.$, 
$\left.1.05 P_{L 0}\right]$ and $\left[0.95 Q_{L 0}, 1.05 Q_{L 0}\right]$ respectively, where $P_{L 0}$ and $Q_{L 0}$ are the deterministic load condition in Appendix.

Applying the proposed method, two electromechanical swings modes with smallest damping ratios are selected and their interval damping ratios are solved. The interval damping ratio and its calculation time are shown in Table 4.

Table 4

Interval damping ratio and computing time of New England system

\begin{tabular}{|c|c|c|}
\hline Mode & Interval Damping Ratio & Calculation Time (unit: second) \\
\hline 1 & {$[0.0382,0.0394]$} & 40.67 \\
\hline 2 & {$[0.0409,0.0428]$} & 40.14 \\
\hline
\end{tabular}

Take the weakest mode for example, the procedure to solve the interval damping ratio is shown in Fig. 5. Meanwhile, the load states corresponding to the interval damping ratios are obtained. When the weakest damping ratio is at the upper limit, the active and reactive power of load $3,4,7,8,15,16$, $18,25,26,27$ are at their upper limits, while the active power of load 12, 20, 24, 28, 29 are not at their interval limits.

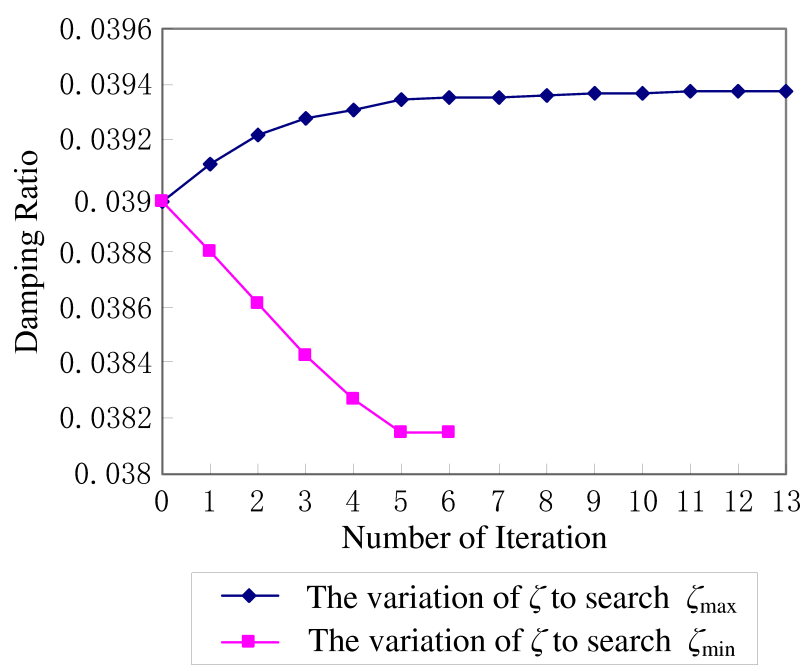

Fig. 5. Searching procedure of the interval damping ratio for New England system

\section{Conclusions}

This paper builds a nonlinear damping ratio optimization model for analyzing small-signal stability under interval load. An effective successive linear programming method is proposed to solve this problem. By using this method, the upper and lower limits of interval damping ratio can be solved and the interval distribution of the damping ratio can be determined, as well as the load states at the limits of the interval damping ratio. The proposed method is tested on two examples. Computation results reveal that load variations have a great impact on damping of oscillation modes. The interval damping ratio supplies distributions of damping modes and assists in taking measures to improve them when the load is interval uncertainty.

\section{Appendix}

Figure 1 shows the 4-machine system one-line diagram where bus 1 is considered to be the slack bus. Each machine is described by the sixth order model in the following order of state-variables $\omega, \delta, E_{q}^{\prime}, E_{q}^{\prime \prime}, E_{d}^{\prime}, E_{d}^{\prime \prime}$. The deterministic operation condition is shown in Table A1. The bus 7 and bus 9 are the load buses.

Table A1

Deterministic operation condition of 4-machine system

\begin{tabular}{ccccc}
\hline \hline Node & $P(\mathrm{MW})$ & $Q$ (MVar) & Voltage(p.u.) & Angle $\left({ }^{\circ}\right)$ \\
\hline 2 & 610 & 104.2 & 1.010 & 1.8 \\
\hline 3 & 660 & 165.7 & 1.030 & 20.5 \\
\hline 4 & 500 & 189.4 & 1.010 & 9.7 \\
\hline 7 & 872 & 100 & 0.970 & -8.6 \\
\hline 9 & 1041 & 100 & 0.951 & -2.1 \\
\hline
\end{tabular}

In New England 10-machine 39-bus system, bus 31 is the slack node and buses numbered from 30 to 39 are generator buses. All machines are described by sixth order models. The deterministic operation condition is shown in Table A2.

Table A2

Deterministic operation condition of New England system

\begin{tabular}{ccccc}
\hline \hline Node & $P(\mathrm{MW})$ & $Q$ (MVar) & Voltage(p.u.) & Angle $\left(^{\circ}\right)$ \\
\hline 3 & 297 & 2.4 & 1.024 & -18.6 \\
\hline 4 & 535 & 184 & 0.993 & -18.5 \\
\hline 7 & 257 & 84 & 0.984 & -18.1 \\
\hline 8 & 583 & 176 & 0.984 & -18.9 \\
\hline 12 & 9 & 88 & 0.990 & -14.3 \\
\hline 15 & 353 & 153 & 1.005 & -18.3 \\
\hline 16 & 339 & 32.3 & 1.022 & -17.2 \\
\hline 18 & 155 & 30 & 1.024 & -18.7 \\
\hline 20 & 762 & 103 & 0.988 & -15.5 \\
\hline 21 & 287 & 115 & 1.024 & -14.8 \\
\hline 23 & 230 & 84.6 & 1.039 & -10.4 \\
\hline 24 & 325 & 10 & 1.023 & -17.1 \\
\hline 25 & 215 & 47.2 & 1.054 & -14.9 \\
\hline 26 & 142 & 17 & 1.048 & -16.5 \\
\hline 27 & 285 & 75.5 & 1.032 & -18.4 \\
\hline 28 & 218 & 27.6 & 1.048 & -13.3 \\
\hline 29 & 300 & 26.9 & 1.048 & -10.6 \\
\hline 31 & 10 & 4.6 & 0.982 & 0 \\
\hline 39 & 1246 & 250 & 1.030 & -21.9 \\
\hline & & & &
\end{tabular}

\section{REFERENCES}

[1] L. Xu and S. Ahmed-Zaid, "Tuning of power system controllers using symbolic eigensensitivity analysis and linear programming", IEEE Trans. on Power Systems 10 (1), 314-322 (1995).

[2] M. Xiao-ming, Z. Yao, G. Lin, and W. Xiao-chen, "Coordinated control of interarea oscillation in the China southern power grid", IEEE Trans. Power Systems 21 (2), 845-852 (2006).

[3] L. Wang and A. Semlyen, "Application of sparse eigenvalue techniques to the small signal stability analysis of large power systems", IEEE Trans.s on Power Systems 5 (2), 635-642 (1990). 
[4] Z. Du, W. Liu, and W. Fang, "Calculation of electromechanical oscillation modes in large power systems using JacobiDavidson method", IEE Proc.-Gener. Transm. Distrib. 152 (6), 913-918 (2005).

[5] R. Różycki and J. Węglarz, "On job models in power management problems", Bull. Pol. Ac.: Tech. 57(2), 147-151 (2009).

[6] K.W. Wang, C.T. Tse, and K.M. Tsang, "Algorithm for power system dynamic stability studies taking account of the variation of load power", Electric Power Systems Research 46 (3), 221-227 (1998).

[7] C.Y. Chung, K.W. Wang, C.T. Tse, and R. Niu, "Powersystem stabilizer (PSS) design by probabilistic sensitivity indexes (PSIs)", IEEE Trans. on Power Systems 17 (3), 688-693 (2002).

[8] K.W. Wang, C.Y. Chung, C.T. Tse, and K.M. Tsang, "Probabilistic eigenvalue sensitivity indices for robust PSS site selection”, IEE Proc.-Gener. Transm. Distrib. 148 (6), 603-609 (2001).

[9] J.L. Rueda, D.G. Colomé, and I. Erlich, "Assessment and enhancement of small signal stability considering uncertainties", IEEE Trans. on Power Systems 24 (1), 198-207 (2009).

[10] Z. Wang and F.L. Alvarado, "Interval arithmetic in power flow analysis", IEEE Trans. on Power Systems 7 (3), 1341-1349 (1992).

[11] B. Das, "Radial distribution system power flow using interval arithmetic", Electric Power Systems Research 24 (10), 827-836 (2002).

[12] P. Wu, H. Cheng, and J. Xing, "The interval minimum load cutting problem in the process of transmission network expansion planning considering uncertainty in demand", IEEE Trans. Power Syst. 23 (3), 1497-1506 (2008).

[13] Y.V. Makarov, V.A. Maslennikov, and D.J. Hill, "Revealing loads having the biggest influence on power system small dis- turbance stability", IEEE Trans. on Power Systems 11 (4), 2018-2023 (1996).

[14] V.A. Maslennikov, J.V. Milanovic, and S.M. Ustinov, "Robust ranking of loads by using sensitivity factors and limited number of points from a hyperspace of uncertain parameters", IEEE Trans. Power Syst. 17 (3), 565-570 (2002).

[15] P. Kundur, Power System Stability and Control, McGraw-Hill Press, New York, 1994.

[16] J. Nocedal and S.J. Wright, Numerical Optimization, Springer New York Press, New York, 1999.

[17] O. Alsac, J. Bright, M. Prais, and B. Sttot, "Further developments in LP-based optimal power flow", IEEE Trans. on Power Systems 5 (3), 697-711 (1990).

[18] L. Shengsong, W. Min, and H. Zhijian, "Hybrid algorithm of chaos optimisation and SLP for optimal power flow problems with multimodal characteristic", IEE Proc.-Gener. Trans. Distrib. 150 (5), 543-547 (2003).

[19] R. Gabasov and F.M. Kirillova, "Optimal real-time control for dynamical systems under uncertainty”, Bull. Pol. Ac.: Tech. 55 (1), 7-13 (2007).

[20] A. J. Jordan, "Linearization of non-linear state equation", Bull. Pol. Ac.: Tech. 54 (1), 63-73 (2006).

[21] C.Y. Chung, K.W. Wang, C.K. Cheung, C.T. Tse, and A.K. David, "Machine and load modeling in large scale power industries", Dynamic Modeling Control Applications for Industry Workshop, IEEE Industry Applications 1, 7-15 (1998).

[22] W.KW Chung, C.T Tse, "Multimachine eigenvalue sensitivities of power system parameters", IEEE Trans. on Power Systems 15 (2), 741-747 (2000).

[23] L. Gang, "Small signal stability analysis and object oriented software developing", PhD Thesis, Shanghai Jiaotong University, Shanghai, 2000. 